\title{
Stochastic Programming for Optimal Decision Making through Scaling Measures
}

\author{
${ }^{1} \mathrm{P}$. Tirupathi Rao, ${ }^{2}$ Kiran Kumar Paidipati and ${ }^{3} \mathrm{C}$. Umashankar \\ ${ }^{1}$ Associate Professor, Dept. of Statistics, Pondicherry University, Puducherry -605014, India \\ Email: drtrpadi@gmail.com \\ ${ }^{2}$ Research Scholar, Dept. of Statistics, Pondicherry University, Puducherry -605014, India \\ Email: kirankumarpaidipati@gmail.com \\ ${ }^{3}$ Professor, Dept. of S.Q.C. \& O.R., Rayalaseema University, Kurnool, A.P. India \\ Email: drcuma.shankar@gmail.com
}

\begin{abstract}
In this paper, a stochastic programming problem for scaling measures similar to Likert's format is developed. The objective function is formulated with a view of maximizing the expected decision making score. The constraints are designed with minimum expected decision score and with minimum targeted precision. The very objective of this problem is to find the decision variables of finding the optimal handled number of assignments by a manager in different categories. While developing the programming problem, the statistical measures such as mean and variances of decision scores are derived from the developed stochastic models based on bivariate stochastic processes as a result of spread sheet experimentation. Sensitivity analysis is carried out with the numerical outputs after solving the derived NLPP using a mathematical software LINGO.
\end{abstract}

\section{KEY WORDS:}

Decision Scores, Scaling Measures, Stochastic programming problem, Bivariate stochastic processes, Stochastic Modeling

\section{ACADEMIC DISCIPLINE AND SUB-DISCIPLINES}

Mathematical Statistics, Operations Research;

\section{SUBJECT CLASSIFICATION}

Operational Management, Stochastic Modeling

\section{TYPE (METHOD/APPROACH)}

semi theoretical approach of Operational Research and applications of measuring scales

\section{Council for Innovative Research}

Peer Review Research Publishing System

Journal: International Journal of Management \& Information Technology

\author{
Vol. 6, No. 1 \\ editor@cirworld.com \\ www.cirworld.com, member.cirworld.com
}




\section{INTRODUCTION:}

Managerial decisions are often based on common sense and environmental issues. Quantification through spread sheets, statistical scaling measures, mathematical formulae, stochastic models, etc are some prime methods that are being used in decision making protocols. Assessment and evaluation of the problem under study has to be handled with the proper parameters of the concerned population. Theoretical development of quantification models for exploring the parameters of the population using the stochastic processes gaining importance as these modeling is the suitable option for dealing the uncertainty assumptions. Expedition of $\mathrm{Bi}$-variate stochastic processes with spread sheet quantification experiments have been considered to the proposed model development. Derivation of mathematical relations for probability distributions, joint moments, marginal moments, measures of discrete statistics like mean, variance, skewness, kurtosis and similar formulae is the real objective of this work. Development of decision support systems through this study is more effective in many of the domains such as Optimal medical care protocols, Managerial performance studies, Device effectiveness measurements, etc. This study has the scope of formulating more generalized scaling measures, Derivations of assessment devices, protocols of inferential studies, Designing of Optimization tools, Development of Decision Support Systems and many more of similar objectives. Proper Perceptions on a research problem are feasible through their suitable measures and competent analytical view of them.

Measurement consists of two basic processes called conceptualization and operationalization with the objectives like determining the levels of measurement, Measuring reliability and validity. Quantification of qualitative characteristics has given big breakthrough in all most all research studies. The quantification phenomena is the land mark research investigation in pursuing decision-making issues. The research findings become statistical oriented, as they are indispensible for rational understanding of the problem under study. The applications of statistical tools are expanding their horizons in expedition of truth and evidence in the thirst for knowledge. Visualizing the interiors of the data is possible through the relevant data structures. And model has to be considered as anatomy of a data patterns. The technology of statistical computing become the order of the day. Hence the computing machines have to be monitored and governed by suitable theoretical models. Measuring the quantified values in managerial decisions have to equipped with relevant models. Extraction of data hidden knowledge and business intelligence is a partial part of data modeling.

Therese Hoff Macan (1994) discussed the theoretical framework on effective time management with empirical examination and tested a process model of time management to study the results in increased job performance and satisfaction where the employees performing variety of jobs on several types of scales. Richard et. al. (1996), reviewed some general properties of mixtures in stochastic processes and statistics utilized in climate applications and described the issues applied in certain types of statistical down scaling. Caryl $E$ et.al.(1998) studied and evaluated the reliability and validity of the Investment Model Scale based on an instrument designed to measure the commitment levels, dependencesatisfaction level, quality of alternatives, and investment size. James Risbey et.al. (1999), presented a framework for viewing agricultural adaptation and emphasizing the multiple spatial, temporal scales applied in climate studies. Mather (1999) presented a framework to use and developing spreadsheet based business models, which are flexible and more useful through which efficiency of modeling can be achieved. Elke U. Weber et.al. (2002) presented psychometric scale that assesses risk taking in five content domains namely financial decisions, health/safety, recreational, ethical, and social decisions. Guy gable et.al. (2003) presented a validated measurement model and instrument for assessing enterprise systems success from multiple perspectives by employing 27 measures of the four dimensions of enterprise systems success (ESS) namely information quality, system quality, individual impact, and organizational impact. Thin-Yin Leong et.al. (2008) proposed Modeling of business concerns with spreadsheet without use of mathematics and statistics as the basic skills in management science and the pedagogical challenges. Yuh-Yuan Guh et.al. (2009) presented an additive scale model for the Analytic Hierarchy Process (AHP) that suits the decision problem using a linear preference comparison, which relates to mathematical denotation, axiom, transitivity and numerical analysis for the additive scale model of AHP. R Brian Haynes, Nancy L Wilczynski (2010) has provided a decision-maker-researcher partnership model for systematic knowledge translation and presented the data using descriptive summary measures, including proportions for categorical variables and means for continuous variables. Ruth et.al. (2013) studied the Psychometric Evaluation of the Surrogate Decision Making (SDM) Self-Efficacy Scale, which can be used as an assessment instrument as well as an outcome measure for interventions. U.B. Baizyldayeva et.al. (2013) proposed multi criteria decisionmaking procedural scales for handling the complex classification of software represented in different data formats.

The above literature has given much emphasis on making use of psychometric and related measurement scales with the help of statistical and computational methods. But there a little evidence on measuring the decision making items and its accumulated impact with suitable scaling formats. No attempt has been made on modeling the likert and Symantec scales either with mathematical or with statistical methodologies. Keeping this gap of research in mind we have developed a stochastic model on measurement of overall decision making score. The variance of the decision score is also derived. Further, the derived statistical measures are used to formulate the programming problems with the objectives of maximizing the overall decision making score. The prime objective of this work is to identify the Bivariate stochastic process for measuring the decision score. It will provide the models of scaling measures by means of stochastic processes. The study is covered in two phases. In phase one, we develop the Bi-variate stochastic process for decision item and scale measure; separate uni-variate stochastic processes for decision item and scale measures. In phase two, we have formulated an optimization programming problem and analyzed the behaviour. 


\section{STOCHASTIC MODEL AND OPTIMIZATION PROBLEM}

Modeling the optimization programming problems to suit manager's requirement can be well planned with various statistical measures. For instances the average decision making score is will help to measure the effectiveness of decision making process. In this section we have formulated some optimization programming problems for exploring the optimal decision variables. In this model the computations are made in view of considering the Decision items. It is assumed that all the decision items are included in the process of decision making. As there are ' $m$ ' decision items and each measured on ' $n$ ' points of Likert scale, the model is developed with the following assumptions.

Let there be ' $m>0$ ' number of decision items that are measured on ' $\mathrm{k}>0$ ' point scale. The decision scores are calculated on each of the items and sum up the all decision items to measure the total score. The Bivariate frequency distributions of empirical data may be used to get the relative frequency/ probability distributions. While getting the probability distributions, we have considered two cases such as (i) All the decision items are equally likely for being in the decision making process. (ii) different decision items have different preferential weight ages for their being in the probability distribution. Model behaviour is observed with a hypothetical data set and conducted the sensitivity analysis.

Impact level of each decision item is scaled on ' $n$ ' points among which any one unit of scale will be considered as suitable. This scale is discrete in nature and taking the integer values such that the score value of $i^{\text {th }}$ decision item lies between 1 and $\mathrm{n}$; the selection of decision item is based on the discretion of the decision maker, purely depends on the choice of him. The decision maker may choose either all the decision items in the list or he may confine to a few decision items, which he feel rational.

Let 'i' be the type of decision item, considered for decision making under study. It is a Decision item (variable), i=1,2,...m. Where ' $m$ ' is the total number of available decision options. Let ' $j$ ' be the score point of the decision item, usually rated on 'j' points scale for $j=1,2,3, \ldots . n$, where ' $n$ ' is maximum score point that a decision item can score. Let T be the Total score then the related statistical measures say central moments can be obtained accordingly

Let $P_{i j}$ be Probability of Choosing the $i^{\text {th }}$ decision item with $j^{\text {th }}$ score point. such that $P_{i j}=0$ when $i^{\text {th }}$ decision item with $j^{\text {th }}$ score point was not selected, where as $P_{i j}=1$, when $i^{\text {th }}$ decision item with $j^{\text {th }}$ score point was selected. Here we have, $\sum_{j=1}^{n} P_{i j}=P_{i}=1$ for all $\mathrm{i}=1,2, \ldots . . \mathrm{m} . \quad \sum_{i=1}^{m} P_{i}=\sum_{i=1}^{m} 1=m, \quad$ With the above considerations, The mean and variance of

Decision scores are as shown below.

The average decision score is $E(T)=\sum_{i=1}^{m} \sum_{j=1}^{n} i . j \cdot p_{i j}$;

The variance of the Decision score is $V(T)=\sum_{i=1}^{m} \sum_{j=1}^{n} i . j^{2} p_{i j}-\left(\sum_{i=1}^{m} \sum_{j \neq k=1}^{n} i . j p_{i j}\right)^{2}$

The objective of this programming problem is to explore the decision variables such as the number of different decision assignments belongs to various categories. This sort of operational management is required to the agencies where they develop the decision support systems for deciding the optimal performing number of assignments.

Let the organization have ' $k$ ' types of assignments based on which the decision making exercise such as finding the decision variables like optimal number of assignments is to be done, where $k=1,2,3, \quad, r ; r$ is the maximum number of different types of assignments.

Let $X_{k}$ be the number of decision assignments in the $k^{\text {th }}$ category of an organization.

Let $C_{k}$ be the expected decision score on $k^{\text {th }}$ category per assignment.

Let $Z=\sum_{k=1}^{r} C_{k} X_{k}$ which implies $Z=\sum_{k=1}^{r}\left(\sum_{i=1}^{m} \sum_{j=1}^{n} i . j \cdot p_{i j}\right) * X_{k}$ be the total expected decision score on all ' $r$ ' types of decision assignments. Since $Z$ is the expected decision score, the objective to maximize $Z$.

Let $T_{k}$ be the expected decision score for one assignment in the kth category. The total expected decision score for $X_{k}$ assignments in the $k^{\text {th }}$ category is $T_{k}{ }^{*} X_{k}$. Therefore the overall expected score for all types of ' $r$ ' assignments is $\sum_{k=1}^{r} T_{k} * X_{k}$. Usually the organization shall handle the decision making activities based on the expected score of each category of assignments and also on overall expected score of ' $r$ ' assignments. Let $S_{k}$ be the minimum targeted decision score based on which the kth category of assignment is to be assessed, then the expected score should not be less than the specified score. Therefore, by considering the above issues, the constraints on the targeted score may be 
$\mathrm{E}\left(\mathrm{T}_{\mathrm{k}^{\star}} \mathrm{X}_{\mathrm{k}}\right) \geq \mathrm{S}_{\mathrm{k}} ;$ which implies that $\sum_{i=1}^{m} \sum_{j=1}^{n} i \cdot j \cdot p_{i j} * X_{k} \geq S_{k}$. for $\mathrm{k}=1,2,3, \mathrm{r} ; \quad$ Further, we consider a constraint that the overall decision score based on all 'r' categories. Let $\sum_{k=1}^{r} S_{k}=S$, then the constraint is $\sum_{k=1}^{r}\left(\sum_{i=1}^{m} \sum_{j=1}^{n} i \cdot j \cdot p_{i j} * X_{k}\right) \geq S$

While achieving the objective, the decision maker has to observe the volatility score on decision making processes. Let $V\left(T_{k}\right)$ be the variability of ' $k^{\text {th' }}$ type of one assignment. Then the variability for $X_{k}$ number of assignments is $V\left(T_{k^{*}} X_{k}\right)$. Further, a healthy decision making procedure of kth type requires the maximum restriction on the variability for $k^{\text {th }}$ category of assignments. Let $\mathrm{V}_{\mathrm{k}}$ be the maximum desired variability for $\mathrm{X}_{\mathrm{k}}$ number of assignments in the $\mathrm{k}^{\text {th }}$ category.

Therefore the overall variability for the $k^{\text {th }}$ category should not exceed the specified level, i.e. $V_{k} . H e n c e ~ V\left(T_{k}{ }^{*} X_{k}\right) \leq V_{k}$, which implies that $\left(\sum_{i=1}^{m} \sum_{j=1}^{n} i . j^{2} p_{i j}-\left(\sum_{i=1}^{m} \sum_{j \neq k=1}^{n} i . j p_{i j}\right)^{2}\right) * X_{k} \leq V_{k}$ for $\mathrm{k}=1,2, \ldots . r . \quad$ Further, we consider a constraint that the overall variability on decision score based on all ' $r$ ' categories.

Let $\sum_{k=1}^{r} V_{k}=V$, then the constraint is $\sum_{k=1}^{r}\left(\sum_{i=1}^{m} \sum_{j=1}^{n} i . j^{2} p_{i j}-\left(\sum_{i=1}^{m} \sum_{j \neq k=1}^{n} i . j p_{i j}\right)^{2}\right) * X_{k} \leq V$

The decision variables under study are non negative. Hence $X_{k} \geq 0$

Therefore the overall optimization programming problem is

Maximize $Z=\sum_{k=1}^{r}\left(\sum_{i=1}^{m} \sum_{j=1}^{n} i \cdot j \cdot p_{i j}\right) * X_{k}$

Subject to the constraints

$\sum_{i=1}^{m} \sum_{j=1}^{n} i . j \cdot p_{i j} * X_{k} \geq S_{k}$ for $\mathrm{k}=1,2, \ldots$.

$\left(\sum_{i=1}^{m} \sum_{j=1}^{n} i . j^{2} p_{i j}-\left(\sum_{i=1}^{m} \sum_{j \neq k=1}^{n} i . j p_{i j}\right)^{2}\right) * X_{k} \leq V_{k}$ for $\mathrm{k}=1,2, \ldots \mathrm{r}$;

$\sum_{k=1}^{r}\left(\sum_{i=1}^{m} \sum_{j=1}^{n} i \cdot j \cdot p_{i j} * X_{k}\right) \geq S$

$\sum_{k=1}^{r}\left(\sum_{i=1}^{m} \sum_{j=1}^{n} i \cdot j^{2} p_{i j}-\left(\sum_{i=1}^{m} \sum_{j \neq k=1}^{n} i . j p_{i j}\right)^{2}\right) * X_{k} \leq V$

And $X_{k} \geq 0$; for $k=1,2, \ldots$;

\section{NUMERICAL ILLUSTRATION AND ANALYSIS:}

The above three model is analyzed with a simulated numerical data sets. Extraction of decision variables, namely number of optimal decision assignments in each category can be obtained. All the above problems are comes under the category of Linear Programming Problems. Initial purpose of this chapter is to calculate the optimal function values and the values of decision variables. The next objective is to analyze the patterns of objective function values with its influencing factors such as $\mathrm{N}_{\mathrm{i}}, \mathrm{N}, \mathrm{P}_{\mathrm{ij}}, \mathrm{m}, \mathrm{n}, \mathrm{k}, \mathrm{r}, \mathrm{V}_{\mathrm{k}}, \mathrm{V}, \mathrm{S}_{\mathrm{k}}, \mathrm{S}, \mathrm{T}_{\mathrm{k}}, \mathrm{T}, \mathrm{W}_{\mathrm{i}}, \mathrm{C}_{\mathrm{k}}$. The study will provide detail understanding on the objective functions. The study also provides the guiding aspects on the constraints about their feasibilities and other related restrictions.

From Table-1 and Graph 1 , it is observed that the objective value is an increasing function of $V_{1}, V_{2}, V_{3}, V_{4}$, and $V_{5}$ when other parameters are constant. Further the decision variable $X_{1}, X_{2}, X_{3}, X_{4}$, and $X_{5}$ are increasing functions of $V_{1}, V_{2}, V_{3}, V_{4}$ and $V_{5}$ respectively and they are invariant with other complementary values of the respective Vi's for $i=1,2,3,4,5$. 
ISSN 2278-5612

Table-1: Values of Decision variables and the optimal value of the objective function for varying values of other parameters

\begin{tabular}{|c|c|c|c|c|c|c|c|c|c|c|c|c|c|c|c|c|c|c|c|c|}
\hline \multicolumn{5}{|c|}{ Matrix } & $S_{1}$ & $\mathrm{~S}_{2}$ & $\mathrm{~S}_{3}$ & $\mathrm{~S}_{4}$ & $\mathrm{~S}_{5}$ & $\mathrm{~V}_{1}$ & $V_{2}$ & $\mathrm{~V}_{3}$ & $\mathrm{~V}_{4}$ & $V_{5}$ & $X_{1}$ & $X_{2}$ & $x_{3}$ & $X_{4}$ & $X_{5}$ & Z \\
\hline 0 & 0 & 0 & 1 & 0 & 1 & 2 & 1 & 1 & 1 & 2 & 5 & 8 & 6 & 7 & 12.32 & 12.35 & 12.37 & 12.35 & 12.36 & 2470.15 \\
\hline 0 & 0 & 0 & 0 & 1 & & & & & & 9 & & & & & 12.38 & 12.35 & 12.37 & 12.35 & 12.36 & 2472.31 \\
\hline 1 & 0 & 0 & 0 & 0 & & & & & & 13 & & & & & 12.41 & 12.35 & 12.37 & 12.35 & 12.36 & 2473.54 \\
\hline 0 & 1 & 0 & 0 & 0 & & & & & & 18 & & & & & 12.45 & 12.35 & 12.37 & 12.35 & 12.36 & 2475.08 \\
\hline 0 & 0 & 1 & 0 & 0 & & & & & & 21 & & & & & 12.47 & 12.35 & 12.37 & 12.35 & 12.36 & 2476 \\
\hline 0 & 0 & 0 & 1 & 0 & 1 & 2 & 1 & 1 & 1 & 2 & 10 & 8 & 6 & 7 & 12.32 & 12.38 & 12.37 & 12.35 & 12.36 & 2471.69 \\
\hline 0 & 0 & 0 & 0 & 1 & & & & & & & 15 & & & & 12.32 & 12.42 & 12.37 & 12.35 & 12.36 & 2473.23 \\
\hline 1 & 0 & 0 & 0 & 0 & & & & & & & 20 & & & & 12.32 & 12.46 & 12.37 & 12.35 & 12.36 & 2474.77 \\
\hline 0 & 1 & 0 & 0 & 0 & & & & & & & 25 & & & & 12.32 & 12.5 & 12.37 & 12.35 & 12.36 & 2476.31 \\
\hline 0 & 0 & 1 & 0 & 0 & & & & & & & 30 & & & & 12.32 & 12.54 & 12.37 & 12.35 & 12.36 & 2477.85 \\
\hline 0 & 0 & 0 & 1 & 0 & 1 & 2 & 1 & 1 & 1 & 2 & 5 & 12 & 6 & 7 & 12.32 & 12.35 & 12.4 & 12.35 & 12.36 & 2471.39 \\
\hline 0 & 0 & 0 & 0 & 1 & & & & & & & & 16 & & & 12.32 & 12.35 & 12.43 & 12.35 & 12.36 & 2472.62 \\
\hline 1 & 0 & 0 & 0 & 0 & & & & & & & & 20 & & & 12.32 & 12.35 & 12.46 & 12.35 & 12.36 & 2473.85 \\
\hline 0 & 1 & 0 & 0 & 0 & & & & & & & & 24 & & & 12.32 & 12.35 & 12.49 & 12.35 & 12.36 & 2475.08 \\
\hline 0 & 0 & 1 & 0 & 0 & & & & & & & & 28 & & & 12.32 & 12.35 & 12.52 & 12.35 & 12.36 & 2476.31 \\
\hline 0 & 0 & 0 & 1 & 0 & 1 & 2 & 1 & 1 & 1 & 2 & 5 & 8 & 9 & 7 & 12.32 & 12.35 & 12.37 & 12.38 & 12.36 & 2471.08 \\
\hline 0 & 0 & 0 & 0 & 1 & & & & & & & & & 12 & & 12.32 & 12.35 & 12.37 & 12.4 & 12.36 & 2472 \\
\hline 1 & 0 & 0 & 0 & 0 & & & & & . & & & & 15 & & 12.32 & 12.35 & 12.37 & 12.42 & 12.36 & 2472.92 \\
\hline 0 & 1 & 0 & 0 & 0 & & & & & 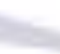 & & & & 18 & & 12.32 & 12.35 & 12.37 & 12.45 & 12.36 & 2473.85 \\
\hline 0 & 0 & 1 & 0 & 0 & & & & & & & & & 21 & & 12.32 & 12.35 & 12.37 & 12.47 & 12.36 & 2474.77 \\
\hline 0 & 0 & 0 & 1 & 0 & 1 & 2 & 1 & 1 & 1 & 2 & 5 & 8 & 6 & 11 & 12.32 & 12.35 & 12.37 & 12.35 & 12.39 & 2471.39 \\
\hline 0 & 0 & 0 & 0 & 1 & & & & & & & & & & 15 & 12.32 & 12.35 & 12.37 & 12.35 & 12.42 & 2472.62 \\
\hline 1 & 0 & 0 & 0 & 0 & & & & & & & & & & 19 & 12.32 & 12.35 & 12.37 & 12.35 & 12.45 & 2473.85 \\
\hline 0 & 1 & 0 & 0 & 0 & & & & & & & & & & 23 & 12.32 & 12.35 & 12.37 & 12.35 & 12.48 & 2475.08 \\
\hline 0 & 0 & 1 & 0 & 0 & & & & & & & & & & 27 & 12.32 & 12.35 & 12.37 & 12.35 & 12.52 & 2476.31 \\
\hline
\end{tabular}




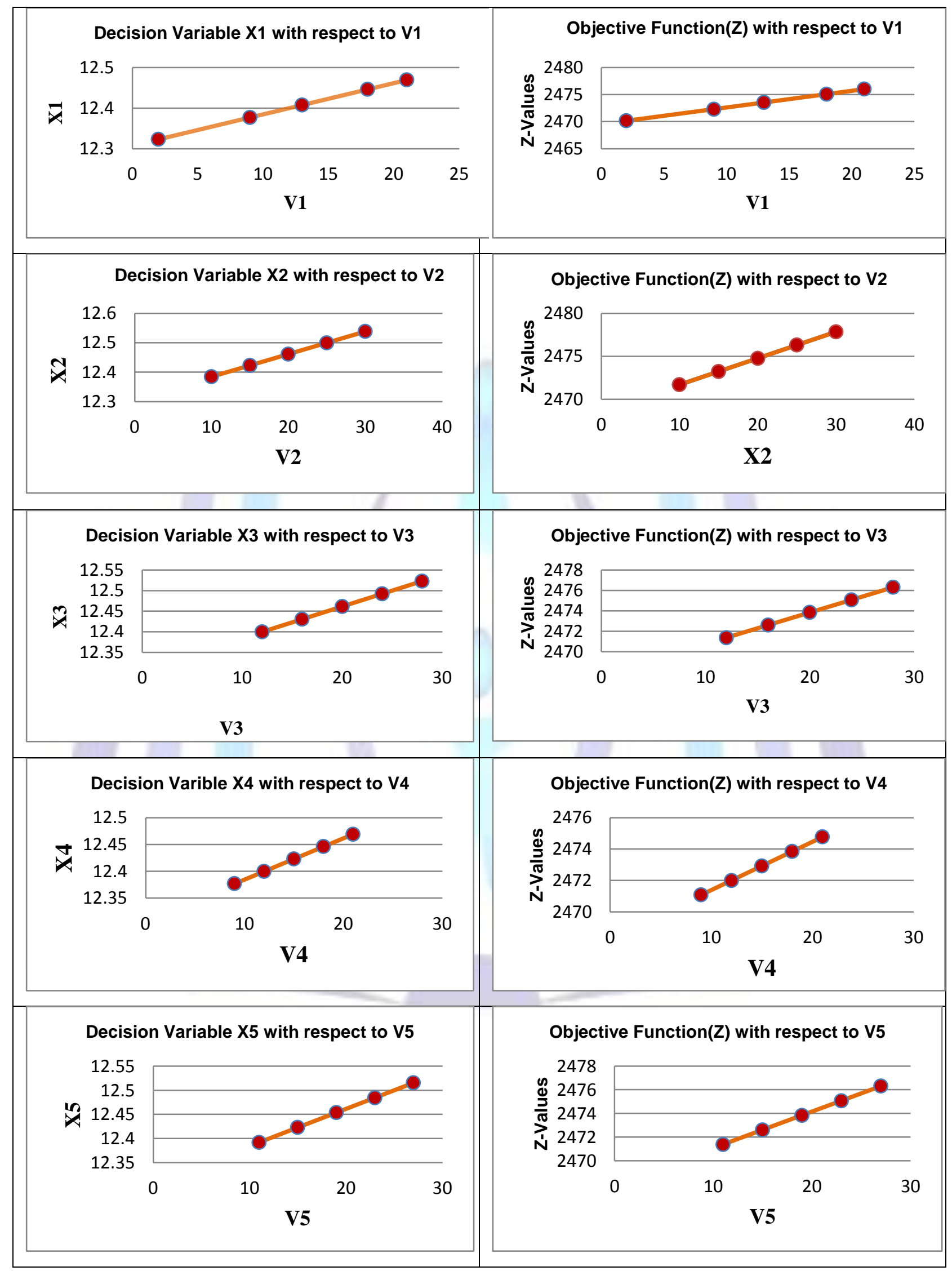




\section{REFERENCES}

[1] Caryl E. Rusbult,O John M. Martz, Christopher R. Agnew (1998), "The Investment Model Scale: Measuring commitment level, satisfaction level, quality of alternatives, and investment size, Personal Relationships, 5 (1998), 357-391.

[2] D Mather (1999), A framework for building spreadsheet based decision models; Journal of the Operational Research Society (1999) 50, 70-74

[3] Elke U. Weber, Ann-Rene' E Blais, Nancy E. Betz, (2002) "A Domain-specific Risk-attitude Scale: Measuring Risk Perceptions and Risk Behaviors”, Journal of Behavioral Decision Making; Vol.15, PP: 263-290 (2002).

[4] Guy G. Gable, Darshana Sedera, and Taizan Chan (2003), Enterprise Systems Success: A Measurement Model; Proceedings Twenty-Fourth International Conference on Information Systems, pages pp. 576-591, Seattle, USA.

[5] James Risbey, Milind Kandlikar And Hadi Dowlatabadi (1999), "Scale, Context, and Decision Making in Agricultural Adaptation to Climate Variability and Change"Mitigation and Adaptation Strategies for Global Change 4:137-165, 1999

[6] R Brian Haynes, Nancy L Wilczynski (2010), "Effects of computerized clinical decision support systems on practitioner performance and patient outcomes: Methods of a decision-maker researcher Partnership systematic review"; Implementation Science 2010, 5:12, http://www.implementationscience.com/content/5/1/12

[7] Richard W.Kaz, Marc B. Carlange (1996), "Mixtures of stochastic processes: application to statistical downscaling"; Climate Research, Vol. 7: 185-193, 1996.

[8] Ruth Palan Lopez, and A.J. Guarino(2013), Psychometric Evaluation of the Surrogate Decision Making SelfEfficacy Scale, Research in Gerontological Nursing • Vol. 6, No. 1, 2013, PP: 71-76,

[9] Therese Hoff Macan (1994), Time Management: Test of a Process Model, Journal of Applied Psychology, 1994, Vol. 79. No. 3, 381-391.

[10] Thin-Yin Leong, Michelle L. F. Cheong (2008), "Teaching Business Modeling Using Spreadsheets" INFORMS Transactions on Education 9(1), pp. 20-34,

[11] U.B. Baizyldayeva, R.K. Uskenbayeva and S.T. Amanzholova (2013), Decision Making Procedure: Applications of IBM SPSS Cluster Analysis and Decision Tree; World Applied Sciences Journal 21 (8): 1207-1212, 2013

[12] Yuh-Yuan Guh, Rung-Wei Po, Kuo-Ren Lou (2009), "An Additive Scale Model for the Analytic Hierarchy Process"; International Journal of Information and Management Sciences; Vol. 20(2009), PP: 71-88

\section{Brief Biography of Authors}

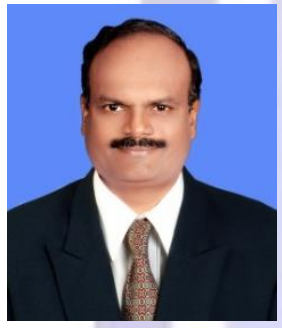

Dr. Tirupathi Rao Padi is presently working as Associate Professor, Dept. of Statistics, Pondicherry University, India. He has 23 years of teaching experience to teach undergraduate, post graduate programs. He has 33 Published research articles in peer reviewed journals, published 9 text books for graduate courses in statistics, completed 2 research projects and 1 is ongoing project, organized 24 academic programs, delivered 50 invited talks at various National and international academic and research forums, Presented 53 research papers, participated in 47 conferences/workshops, guided $3 \mathrm{M}$. Phil. degrees, supervising $4 \mathrm{Ph}$. D. programs. His areas of research include Stochastic Modeling, Applied Operational research, Bio statistics, etc.

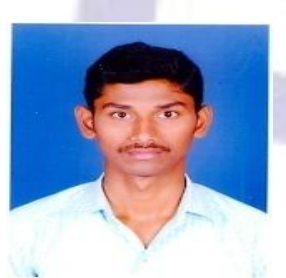

Kiran Kumar Paidipati,is presently Ph.D. full time Research Scholar in the Department of Statistics, Pondicherry University, Puducherry, India. He did his Post Graduation in Statistics at Sri Venkateswara University, Tirupathi, India $\mathrm{He}$ has published two research articles in international journals, participated/ presented research papers in Nine National and International Workshops/conferences/seminars. He qualified in APSET-2012, an eligibility test for Assistant Professor/ Lectureship in the Universities of Andhra Pradesh.

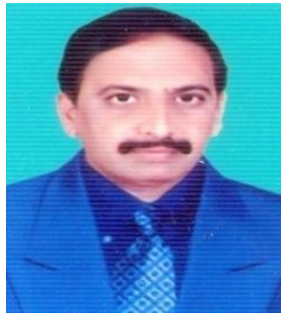

Dr. C. Umashankar is working as Professor, Dept. OR \& SQC, Rayalaseema University, A.P., India. He has 32 Years of teaching and research experience, recipient of best teacher award for 2004 by A.P. Govt. He is the Fellow of Royal Statistical Society, London - 2013, Successfully Guided 18 Ph. D and 8 M. Phil Degrees, Published 50 Research Papers and Presented 100 Research Papers in Different National / International Conferences / Seminars / Symposia And Delivered Expert Lectures. Organized around 20 National / International Workshops and Conferences in Collaboration with University of Delhi, OR Society, ISPS, DRDO, DST, QCI, etc. 